ARTÍCULO DE INVESTIGACIÓN

\title{
EVALUACIÓN DE UN PROTOCOLO DE INTERVENCIÓN EN TERAPIA SISTÉMICA BREVE PARA PERSONAS EXPUESTAS A UN EVENTO ESTRESANTE RECIENTE.
}

\author{
EVALUATION OF A BRIEF SYSTEMIC THERAPY INTERVENTION PROTOCOL FOR \\ PEOPLE WHO HAVE EXPERIENCED A RECENT STRESSFUL EVENT
}

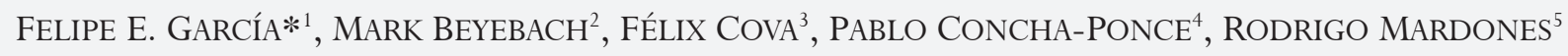

UNIVERSIDAD DE CONCEPCIÓN, CHILE. UNIVERSIDAD PÚBLICA DE NAVARRA, PAMPLONA ESPAÑA Universidad AutónOma de CHILE. Universidad SANTO TOMÁs, CHILE
\end{abstract}

FECHA RECEPCIÓN: 13/03/2021 • FECHA ACEPTACIÓN: 06/05/2021

\begin{abstract}
Para citar este artículo: García, F., Beyebach, M., Cova, F., Concha-Ponce, P., \& Mardones, R. (2021). Evaluación de un protocolo de intervención en Terapia Sistémica Breve para personas expuestas a un evento estresante reciente.

Psychologia, 15(1), 43-55. https:/doi: 10.21500/19002386.5300
\end{abstract}

\section{Resumen}

Los eventos altamente estresantes son sucesos que potencialmente generan consecuencias negativas en las personas afectadas, como síntomas postraumáticos y depresivos. El presente estudio evalúa un protocolo de intervención preventiva, orientado a personas que han vivido un evento altamente estresante reciente, basado en la Terapia Sistémica Breve.

Cuatro terapeutas aplicaron el protocolo a cuatro usuarias. Se entrevistó a estas ocho personas acerca de su experiencia en la aplicación y recepción del protocolo. Mediante escalas de fiabilidad y validez contrastada se evaluaron los cambios pre-post en sintomatología postraumática, sintomatología depresiva, crecimiento postraumático y satisfacción con la vida; también se midió la relación terapéutica. Los resultados muestran apreciaciones positivas en terapeutas y usuarias, el desarrollo de una relación terapéutica adecuada y avances en tres de los cuatro indicadores cuantitativos de cambio. Se concluye que el protocolo está en condiciones de utilizarse en estudios controlados.

1* Departamento de Psiquiatría y Salud Mental, Facultad de Medicina, Universidad de Concepción. ORCID: http://orcid.org/0000-0002-4161-5840, fgarciam@udec.cl Este estudio fue financiado por la beca ANID/Fondecyt 1180134

2 Departamento de Ciencias de la Salud, Universidad Pública de Navarra, Universidad Pública de Navarra. ORCID: http://orcid.org/0000-0002-4490-5915

3 Departamento de Psicología, Facultad de Ciencias Sociales, Universidad de Concepción. https://orcid.org/0000-0002-9290-6875

4 Facultad de Ciencias Sociales y Humanidades, Universidad Autónoma de Chile. ORCID: http://orcid.org/0000-0003-3738-2287

5 Facultad de Ciencias Sociales y Comunicaciones, Universidad Santo Tomás de Chile. ORCID: http://orcid.org/0000-0002-9336-3489 
Palabras claves: protocolo de intervención, prevención, eventos altamente estresantes, sintomatología postraumática, sintomatología depresiva.

\section{Abstract}

Highly stressful events are occurrences that potentially create negative consequences for the affected people, such as post-traumatic and depressive symptoms. This study assesses a preventive intervention protocol, aimed to people who have lived a recent highly stressful event, based on the Brief Systemic Therapy. Four therapists applied the protocol to four female users. These eight persons were interviewed about the experience in relation to the application and reception of the protocol. Through contrasted reliability and validity scales, pre and post changes in posttraumatic symptomatology, depressive symptomatology, posttraumatic growth and satisfaction with life were assessed; the therapeutic relation was also measured. The results show positive appraisals in therapists and female users, the development of a proper therapeutic relation and progress in three out of four quantitative change indicators. It is concluded that the protocol is suitable to be used in controlled studies.

Keywords: intervention protocol, prevention, highly stressful events, posttraumatic symptomatology, depressive symptomatology.

\section{Introducción}

Los eventos altamente estresantes son alteraciones en la historia de vida de un individuo que desafían sus recursos adaptativos (Sutin et al., 2010). Estos eventos, en determinadas circunstancias, pueden alterar la calidad de vida y el bienestar de las personas e incrementar la probabilidad de aparición de dificultades como el trastorno por estrés postraumático (TEPT) y los trastornos depresivos (TD) (Forbes et al., 2020; Van der Kolk, 2014).

De allí el interés de acciones tempranas en personas expuestas a estas condiciones, que pueden atenuar el riesgo de desarrollo de consecuencias que afecten en forma significativa su salud mental posterior.

Por otra parte, también se ha estudiado la capacidad de recuperación y de crecimiento que presentan las personas tras su exposición a eventos estresantes o traumáticos. Si bien la evidencia muestra que algunas personas que experimentan situaciones traumáticas llegan a desarrollar trastornos, en la mayoría de los casos esto no es así (Bonanno \& Diminich, 2013; Bonanno et al., 2011), y algunas incluso son capaces de aprender y beneficiarse de tales experiencias, a lo que se ha llamado crecimiento postraumático (Tedeschi \& Calhoun, 1996). La mantención del bienestar personal o el desarrollo de crecimiento postraumático tras un evento altamente estresante han sido menos estudiados que sus alter- nativas psicopatológicas, y como indicadores de cambio tras intervenciones psicoterapéuticos lo han sido todavía menos, pues estos se han centrado principalmente en la reducción de la sintomatología asociada al malestar.

La mayoría de las intervenciones que se han investigado en relación al tema tienen un marcado sentido correctivo, es decir, se aplican cuando las personas afectadas ya muestran sintomatología avanzada o presentan un diagnóstico psicopatológico; en cambio, las intervenciones preventivas son mucho más escasas (García \& Rincón, 2011). Las intervenciones correctivas que muestran eficacia son en su gran mayoría del ámbito cognitivoconductual; en cambio, en las intervenciones preventivas, además de las propuestas cognitivo-conductuales (i.g. Agorastos et al., 2011), también existe evidencia de que intervenciones centradas en el desarrollo de recursos o habilidades tienen un efecto beneficioso en prevenir malestar en personas que han sido recientemente expuestas a un evento altamente estresante. Entre estas intervenciones se encuentran las derivadas del mindfulness (Boe \& Hagen, 2015), de la psicología positiva (Ochoa et al., 2017) y la terapia sistémica breve (García \& Rincón, 2011). Un aspecto central de estas intervenciones preventivas es disminuir la sintomatología emocional gatillada por la exposición al evento estresante, dado que se ha demostrado que la sintomatología postraumática (SPT) manifestada en los primeros días tras la exposición es un 
fuerte predictor de TEPT (Chen et al., 2015), así como la sintomatología depresiva (SD) pocos días después de un evento es la principal variable predictora de la SD seis meses después (García et al., 2018).

La terapia sistémica breve (TSB) corresponde a un conjunto de procedimientos y técnicas de intervención que pretenden ayudar a los consultantes (individuos, parejas, familias o grupos) a movilizar sus recursos para alcanzar sus objetivos en el menor tiempo posible. Se fundamenta en una perspectiva constructivista centrada en el contexto interpersonal en que se expresan los problemas y sus soluciones, y que promueve una colaboración activa con los usuarios (Herrero de Vega, 2016). Las principales variantes de este modelo lo conforman la terapia breve centrada en soluciones (De Shazer , 1994), la terapia estratégica del MRI de Palo Alto (Weakland et al., 1974) y la terapia narrativa (White \& Epston, 1993).

En una revisión reciente (Eads \& Lee, 2019) se examinaron cinco estudios de resultados de terapia breve centrada en soluciones aplicada a supervivientes de trauma, que incluyeron dos estudios clínicos aleatorizados, dos estudios cuasi experimentales y un estudio pre-post. Considerados en conjunto, los estudios revisados mostraron que este modelo puede ser un tratamiento apropiado para esta población, ya que no se detectaron efectos negativos y en ningún caso fue menos efectivo que los tratamientos habituales, mostrando una reducción de síntomas, efectos positivos sobre variables como la autoestima o las habilidades parentales, además de contribuir al crecimiento postraumático.

Además de estos cinco estudios, en dos ensayos clínicos aleatorizados (ECA) con estudiantes que presentaban malestar emocional (Beauchemin, 2015; Lopes et al., 2014), así como en un estudio cuasiexperimental con mujeres diagnosticadas con cáncer de mama (García \& Rincón, 2011), se documentaron también efectos positivos de la TSB sobre bienestar, estrés, sintomatología depresiva, síntomas postraumáticos y relaciones interpersonales.

A partir de los hallazgos mencionados, en el presente estudio se elaboró un protocolo de intervención que presenta la estructura de una intervención breve de modalidad individual dirigida a personas que reciente- mente se habían visto expuestas a un evento altamente estresante. El propósito principal del presente trabajo es mostrar los resultados de la aplicación piloto de este protocolo, identificando la percepción de las usuarias y terapeutas en relación con el desarrollo y los resultados del proceso de intervención. Secundariamente, se centra en los efectos de la intervención en la sintomatología postraumática, la sintomatología depresiva, el crecimiento postraumático, el bienestar subjetivo y la alianza terapéutica.

\section{Método}

\section{Diseño}

El presente estudio sigue un diseño mixto, cualitativo-cuantitativo. Desde lo cualitativo, se utilizó un método fenomenológico, pues permite un mayor acercamiento a las percepciones y vivencias individuales de los terapeutas y usuarias. Este enfoque intenta considerar los fenómenos tal como son percibidos, experimentados y vividos, centrándose en las experiencias e interpretaciones por parte de las personas que lo viven, enfatizando la experiencia subjetiva (Martínez, 2000). Desde lo cuantitativo, se realizó un estudio de caso intrasujeto de tipo A-B con medidas preintervención, postintervención y seguimiento (Barlow \& Hersen, 1988; Kazdin, 2001).

\section{Participantes}

Participaron cuatro usuarias y cuatro terapeutas. Las usuarias eran personas mayores de 18 años que habían vivido un evento altamente estresante durante los últimos tres meses. Por su parte, las/os terapeutas eran psicólogas/os con experiencia en intervención clínica, tenían formación previa de al menos 100 horas en terapia sistémica y participaron de una capacitación de ocho horas para la aplicación del protocolo. Información sociodemográfica específica de cada uno de ellos se presenta en la Tabla 1. 
Tabla 1. Características sociodemográficas de los participantes

\begin{tabular}{lllll}
\hline \hline C & Rol & Sexo & Edad & Evento \\
\hline T1 & Terapeuta & Hombre & 31 & - \\
T2 & Terapeuta & Hombre & 26 & - \\
T3 & Terapeuta & Mujer & 25 & - \\
T4 & Terapeuta & Mujer & 30 & - \\
Y1 & Usuaria & Mujer & 34 & Enfermedad grave de familiar cercano \\
Y2 & Usuaria & Mujer & 31 & Ruptura matrimonial tras seis años de relación \\
Y3 & Usuaria & Mujer & 56 & Pérdida del hogar por incendio \\
Y4 & Usuaria & Mujer & 52 & Pérdida del hogar por incendio \\
\hline \hline
\end{tabular}

\section{Instrumentos}

Síntomas depresivos: Se utilizó la Escala de Depresión del Centro de Estudios Epidemiológicos (CESD), creada por Radloff (1977), y cuyo uso en población chilena ha mostrado indicadores positivos de validez (Gempp et al., 2004). Consta de 20 ítems, los cuales toman en cuenta la última semana de quien la conteste. Tiene un formato de respuesta tipo Likert que va desde 0 (rara vez) hasta 3 (la mayor parte del tiempo). En población chilena expuestas a un evento estresante ha mostrado altos niveles de consistencia interna $\alpha=0.91$ (García et al., 2018).

Bienestar subjetivo: Se midió con la Escala de Satisfacción Vital (SWLS), creada por Diener et al. (1985), cuyo uso en población de habla hispana ha mostrado positivos indicadores de validez y una consistencia interna de $\alpha$ $=0.81$ (Arias \& García, 2018). Consta de cinco ítems tipo Likert, los cuales van en un rango desde 1 (totalmente en desacuerdo) hasta 7 (totalmente de acuerdo).

Sintomatología postraumática: Se utilizó la escala SPRINT-E, creada por Connor y Davidson (2001). Leiva-Bianchi y Gallardo (2013) observaron un adecuado comportamiento psicométrico de esta escala en población chilena expuesta a un terremoto. La confiabilidad que observaron fue de $\alpha=.92$. Posee 12 preguntas que se responden en una escala Likert desde 0 (nada) hasta 3 (mucho).

Crecimiento postraumático: Se utilizó el Inventario de Crecimiento Postraumático, versión breve (PTGISF), creado por Cann et al. (2010) y validado para su uso en población chilena por García y Wlodarczyk (2015). Está compuesta de 10 ítems que se responden en una escala Likert de seis alternativas, desde 0 (ningún cambio) hasta 5 (un cambio muy importante). En el estudio de García y Wlodarczyk se obtuvo un $\alpha=.94$.

Alianza terapéutica: Se utilizó la Session Rating Scale (SRS) de Duncan et al. (2003), que ha mostrado positivos indicadores de validez en población chilena (Elgueta \& Fantuzzi, 2008). Consiste en una escala que posee cuatro ítems, uno relacionado con el vínculo, otro con los objetivos y temas de la terapia, un tercero con el enfoque del terapeuta y, el último, con la impresión global sobre la sesión. La escala posee afirmaciones polarizadas sobre cada uno de estos ítems (por ejemplo: "No me sentí escuchado, entendido, respetado", "Me sentí escuchado, entendido, respetado") que se ubican en los extremos de una línea horizontal. El paciente hace una marca más cerca de una o de la otra de estas dos afirmaciones para indicar en qué grado evalúa este aspecto. Se aplicó después de cada sesión y se recogió en un sobre sellado de modo que la respuesta no esté sesgada por la presencia del terapeuta.

Entrevista semiestructurada: Se utilizó esta entrevista para indagar en percepciones de usuarias y terapeutas en relación al protocolo tras finalizar la intervención. A las usuarias se les realizó una entrevista individual que incluía preguntas tales como: "¿me puede contar cómo fue la primera sesión?", "¿qué tan útil fue esta intervención para usted?”, "¿qué sugerencias nos puede dar para mejorar la intervención?”. A los terapeutas se les realizó una entrevista grupal que incluía preguntas como: 
“¿podrían describir en sus palabras cómo realizaron la aplicación del protocolo?”, “¿qué sentimientos surgieron durante la aplicación del protocolo?”, “icuál es su opinión sobre el protocolo?”.

\section{Intervención}

El protocolo de intervención preventivo desde la Terapia Sistémica Breve se construyó basándose tanto en la literatura ya descrita en la introducción como en los textos publicados que dan cuenta detalladamente de cómo se desarrolla el proceso terapéutico y cómo se emplean las diversas técnicas de la TSB (Beyebach, 2006; Beyebach \& Herrero de Vega, 2010; García et al., 2016; García \& Schaefer, 2015; REPSSI, 2017). El protocolo considera cuatro sesiones, con una frecuencia de una sesión semanal y una duración de 60 minutos cada sesión (el protocolo detallado puede ser solicitado al autor correspondiente).

La primera sesión se dedica a la construcción de un vínculo terapéutico, la indagación del motivo de consulta y la coconstrucción de objetivos, utilizando para ello una pregunta de escala (De Shazer, 1994), que consiste en invitar a la usuaria a valorar de 1 a 10 en qué punto se encuentra: el 10 sería que ha conseguido todos sus objetivos y el 1 cuando más lejos de ellos ha estado. Al finalizar la sesión, se entrega un mensaje final y se prescribe la tarea-fórmula de la primera sesión (De Shazer, 1994), solicitando al paciente que identifique aspectos personales o en su entorno que están a salvo de las influencias del problema.

La segunda sesión comienza con la revisión de cambios inter-sesiones y de la tarea-fórmula, explorando las áreas libres de problema. A continuación, se utiliza la pregunta de escala para evaluar el nivel de afección percibido por la persona, indagando además excepciones y ampliando percepciones de disminución de la sintomatología relacionada al evento estresante. Luego, se introduce la narrativa de sobreviviente a partir de resúmenes y devoluciones realizados por el terapeuta, poniendo de relieve aquellos aspectos de la narrativa que identifican recursos personales y también excepciones al problema. Con el fin de reconocer y ampliar sus recursos personales, se entrega la tarea de los elogios, en donde la persona debe registrar los elogios que ha recibido en su vida, crea o no en ellos.

La tercera sesión comienza con la revisión de cambios inter-sesiones y de la tarea de los elogios. Como actividad central se inicia el trabajo con el dibujo de árbol de la vida (Ncube \& Denborough, 2008), intervención en donde se representa la historia de vida y experiencias personales, facilitando el reconocimiento de habilidades, recursos, sueños y esperanzas, honrando a las personas importantes y los legados positivos recibidos de ellas. Al finalizar, se prescribe la tarea de la carta desde el futuro, en la cual la consultante debe escribir una carta dirigida de modo ficticio a su yo del presente desde el yo del futuro que ha sido capaz de superar sus dificultades actuales, resaltando qué ha tenido que hacer para llegar a ese momento sin dificultades.

La cuarta y última sesión comienza con la revisión de cambios inter-sesiones y la revisión de la carta desde el futuro. Se continúa con el dibujo del árbol de la vida, concluyendo con una exploración con el consultante sobre las "tormentas de la vida", es decir, dificultades que siguen apareciendo o qué podrían aparecer y cómo cada elemento del árbol puede ayudar a enfrentarlas. Se utiliza la pregunta de escala para evaluar avances desde la primera sesión. Se le pide escribir en sesión una carta a otro consultante que pudiera estar pasando por su mismo problema, de modo de transmitir su propia experiencia de vida y en terapia con el fin de ayudar a esta persona a enfrentar y resolver sus dificultades. Se finaliza con un ritual de cierre en el cual se enmarca su dibujo del árbol de la vida y se le entrega como evidencia del trabajo realizado en la intervención.

\section{Procedimiento}

La selección de los participantes se hizo a través de un muestreo por conveniencia (Martín-Crespo \& Salamanca, 2007). Las usuarias fueron derivadas desde centros de atención psicológica universitaria. El primer contacto se realizó por vía telefónica o directamente se acudió a sus residencias, instancia en las que se les explicó el objetivo del proyecto y en qué consistía su participación. Luego de eso, se procedió a firmar el consentimiento informado de cada usuaria y así dar inicio al proceso de intervención. 
Los terapeutas eran psicólogos titulados en proceso de especialización en terapia familiar. Recibieron una capacitación formal de ocho horas para la aplicación del protocolo.

Los instrumentos de medida se aplicaron una semana antes, una semana después y tres meses después de la intervención. Las entrevistas fueron realizadas dentro de la primera semana después del término de las intervenciones.

Para las entrevistas, se utilizó una modalidad individual para las usuarias y grupal para los terapeutas, que se grabaron en audio en su totalidad. Se realizaron cuatro entrevistas individuales, cuyo promedio de duración fue de 23 minutos y una entrevista grupal de 45 minutos. Se utilizó un guion de entrevista semiestructurada, organizada en temáticas que permitían la introducción de preguntas adicionales para precisar conceptos u obtener más información sobre temas deseados (Hernández, Fernández \& Baptista, 2003). Se siguió un tipo de entrevista focalizado, cuya principal función fue centrar la atención de la persona entrevistada sobre la experiencia concreta que se quería abordar. Posteriormente, se procedió a la transcripción de las respectivas entrevistas, para llevar a cabo con posterioridad el proceso de análisis temático.

El estudio fue aprobado por el Comité Ético Científico de la Universidad Santo Tomas, resolución n. ${ }^{\circ}$ 30/2018.

\section{Análisis de datos}

Los datos cualitativos se analizaron a través de la técnica de análisis temático, la cual consistió principalmente en descomponer los textos en unidades de significado (González \& Cano, 2010), las cuales posteriormente se agruparon según el principio de analogía. Este proceso de análisis se descompuso en los siguientes pasos: 1) se identificaron en los discursos las unidades de análisis; 2) se crearon las categorías emergentes; 3) se elaboró un esquema de codificación; 4) se identificaron las dimensiones y patrones en el discurso de las usuarias y terapeutas entrevistados, y 5) se continuó con el análisis de texto tanto en la entrevista grupal como en las entrevistas individuales. Para la realización de este análisis se utilizó el software del QSR International Nvivo 12 Qualitative Data Analysis Software (QSR International, 2018).
Para los datos cuantitativos se realizó un análisis descriptivo identificando las medias y desviación estándar de las variables. Para la escala de alianza terapéutica se realizó la suma simple de los puntajes obtenidos en cada ítem y en total para todas las sesiones. No se realizaron análisis inferenciales dado el tamaño de la muestra. Se utilizó para ello el programa estadístico SPSS 21.0 (IBM Corp, 2011).

\section{Resultados}

Se presenta, en primer lugar, el análisis cualitativo de las entrevistas realizadas a las usuarias y terapeutas. En segundo lugar, se presenta el análisis cuantitativo de la evaluación pre-post intervención y seguimiento, de las cuatro variables dependientes del estudio.

\section{Análisis cualitativo}

Las temáticas identificadas que aparecieron de forma recurrente se agruparon en las cinco categorías que siguen.

a) Opiniones de las usuarias y terapeutas en relación con su experiencia en terapia.

Al finalizar el proceso, las usuarias manifestaron sentirse desahogadas, con un mayor sentido de bienestar, reconociendo recursos personales y viendo su situación desde otro punto de vista: "me sirvió para desahogarme (...), me sentí bien y relajada" (Y1); "me permitió darme cuenta de cosas que no veía y son mías, sobre todo recursos míos que quizás yo no los veía para nada o los veía de forma negativa (...), me ayudaron a sentirme mejor, a empoderarme de mi misma" (Y2).

Por otro lado, los terapeutas percibieron un cambio positivo en las usuarias desde el inicio del proceso hasta la sesión final: "las personas se fueron bien tranquilas, diferente a como llegaron" (T4).

b) Opinión de las usuarias y de terapeutas en relación con la utilidad del protocolo.

Las usuarias perciben que las sesiones permitieron visualizar los recursos con los que contaban, sentirse más empoderadas y positivas, conversar sus cosas con otras personas y valorar las cosas que la rodean: "tal vez uno no se da cuenta como es uno, (...) el conversar mis cosas, sentir que tengo harta fuerza, ganas de salir adelante" (Y3); "se podría decir que ando más positiva que antes" 
(Y1); "Me di cuenta de cuanto me estiman (...), también me di cuenta de lo mucho que yo valoro también todo lo que tengo, todo lo que he tenido, todo lo que tuve y lo que me rodea actualmente hoy en día. También me sirvió para darme cuenta de que puedo llegar a incentivar, tal vez a otras personas que se encuentren en situaciones difíciles..." (Y2).

En cuanto a las actividades, las usuarias valoraron como significativo la construcción del árbol de la vida: "La del árbol me hizo recordar la vida completa (...), no solamente lo que me está pasando ahora, y con eso las cosas buenas y malas que puedo sacar de ahí" (Y1); "(la última actividad) era la de dibujar un árbol y fijar tus raíces, tu presente, tus características, tus recursos y (...) tus metas, tus sueños, ese me encantó, esas dos últimas sesiones fueron súper significativas para mi” (Y2); “ con el árbol de la vida $(. .$.$) uno se va dando cuenta que hay muchas cosas,$ que uno no es alguien más en la vida, sino que de verdad uno cumple muchas funciones en cuanto a la familia, en la vida" (Y3); "la terapia del arbolito (...) englobó todo, me hizo dar cuenta de mucho (...), de verdad que me fui, como se puede decir, llenita (...) y muy contenta, muy satisfecha, y convencida de que sí había valido la pena el haber estado viniendo a la sesiones" (Y4).

Las usuarias también destacaron la pregunta de escala, que les permitió observar la calidad de su ánimo, expresada en número: “...como empezamos nosotras del uno al diez, yo le decía que consideraba que estaba en el uno porque no quería nada con nada (...), pero ella me hizo ver las cosas buenas y no solo las malas" (Y1).

Las usuarias reconocen la utilidad de las tareas para la casa (tarea fórmula, tarea de los elogios y tarea de la carta desde el futuro) en forma general, sin distinguir claramente unas de otras en sus descripciones: "me sirvió para darme cuenta de lo mucho que tengo" (Y4); "me ayudaron a sentirme mejor, a empoderarme de mi misma" (Y2). Por otro lado, los terapeutas valoraron el cumplimiento de las tareas como favorables para la organización y el desarrollo de las sesiones: "estaba más concentrada en eso y era como súper beneficioso (...), se ordenaba en ese sentido para mí” (T2).

c) Opinión de los terapeutas y usuarias en relación con los obstaculizadores de la intervención y protocolo.

Algunas usuarias indican que antes de haber iniciado las intervenciones presentaban desconfianza por experiencias previas negativas con psicoterapeutas o por dificultades previas para contar aspectos íntimos a terceros: "había tenido anteriormente psicóloga, pero no me había dado la confianza" (Y1); "me cuesta contar mis cosas, decir mis cosas" (Y3). Esto llevó a que tras la primera sesión las usuarias manifestaran algunas interferencias en su encuentro con el psicoterapeuta, aunque también valoraron la sesión como una oportunidad de expresar emociones: "fui a ver (al psicoterapeuta) por un tema en específico y tener que hablarlo con alguien que no conozco quizás me frenó un poco, me costó un poco que fuera más fluida la conversación..." (Y2); “(hubo) mucho llanto, pero después tranquilidad” (Y1).

Algunas usuarias presentaron dificultades para reflexionar sobre los aspectos positivos de su vida o de su experiencia reciente: “... pero esto de ver las cosas positivas de lo que me había pasado, que como esa fue como difícil, eh, porque claro, hay cosas positivas, pero no sé si las quería ver en eso momento" (Y2).

También se visualizaron algunas dificultades en relación con las tareas, como la falta de tiempo, comprensión de las mismas o retroalimentación sobre su correcta ejecución: “...no entendí como hacerla (...) me la había explicado, pero como el tiempo y a donde no ando como pendiente" (Y1); "le costaba hacer las actividades para ella era como el deber de poder cumplir con lo que se había comprometido" (T3).

Dentro de las percepciones de las/los terapeutas en relación con la estructura o claridad del protocolo, se identificaron sesiones con muchas actividades o instrucciones poco claras: "yo decía ¿y ahora que viene? Hacer las preguntas escala, iqué no se me vaya a olvidar que hay que hacer la tarea!, entonces, eso me hacía desconcentrarme de escuchar las palabras de ella" (T2).

d) Elementos facilitadores identificados en el discurso por terapeutas y usuarias durante el desarrollo de las sesiones de intervención.

Las usuarias percibieron facilidad para vincularse con sus terapeutas: “(...) me generó confianza ella [risas] me pude abrir, entonces eso fue bueno para mí, obviamente" (Y2); "me sentí no con una persona ajena, (sino) como alguien conocido" (Y1). Por otro lado, este buen vínculo también es observado por los terapeutas: "se generó bonita alianza y todo, y resulto ser súper positivo" (T2). 
Respecto a la flexibilidad del protocolo, algunos terapeutas se animaron a utilizar otras técnicas del enfoque como el uso de metáforas, cuando coincidía con el discurso del usuario: "yo sí hice una metáfora, porque en verdad era como súper ad hoc al tema" (T3). A su vez, si una tarea no era hecha en casa, el terapeuta la incluía como actividad en la siguiente sesión: "la última fue la única que no hice, que escribiera una carta, pero ella me hizo hacer el ejercicio en sesión para que pudiéramos trabajar con el árbol de la vida" (Y2).

e) Sugerencias de las usuarias y terapeutas para mejorar la intervención.

Las usuarias sugieren un mayor número de sesiones: "yo diría sí, que fueran más sesiones, pero de- pendiendo de la persona" (Y1); "los pacientes no entendemos el tema de la brevedad y necesitamos una o dos sesiones más" (Y2). También surge una sugerencia de dar más tiempo para la ejecución de alguna de las actividades: “Necesitaba más tiempo para el árbol” (Y2).

\section{Análisis cuantitativo}

Se realiza una evaluación de las cuatro variables dependientes del estudio, tanto antes de la intervención como después de ella, y tres meses después, en el seguimiento. Los resultados por cada usuario se observan en la Tabla 2 y las medias se presentan en la Tabla 3.

Tabla 2. Puntajes totales obtenidos por cada usuario en las variables de estudio, en las evaluaciones pre, post y seguimiento

\begin{tabular}{llllllllllllll}
\hline & SPT & \multicolumn{1}{c}{ SD } & \multicolumn{3}{c}{ CPT } & \multicolumn{1}{c}{ SV } \\
\hline & Pre & Post & Seg & Pre & Post & Seg & Pre & Post & Seg & Pre & Post & Seg \\
Y1 & 22 & 16 & 20 & 30 & 39 & 36 & 27 & 37 & 29 & 11 & 13 & 7 \\
Y2 & 22 & 9 & 3 & 25 & 12 & 3 & 26 & 34 & 36 & 23 & 26 & 28 \\
Y3 & 22 & 19 & 18 & 31 & 23 & 24 & 14 & 44 & 37 & 25 & 29 & 33 \\
Y4 & 31 & 25 & 17 & 38 & 29 & 24 & 37 & 46 & 40 & 29 & 27 & 27 \\
\hline \hline
\end{tabular}

Nota: SPT: Sintomatología postraumática; SD: Sintomatología depresiva; CPT: Crecimiento postraumático; SV: Satisfacción con la vida.

Se observa en la Tabla 2 cómo los usuarios en general van disminuyendo en sus niveles de sintomatología postraumática y depresiva a medida que avanza el tiempo, a excepción de Y1, afectado por la enfermedad grave de un familiar cercano, que disminuye la SPT en el post, pero luego vuelve a subir en el seguimiento y aumenta su SD en el post y luego declina ligeramente en el seguimiento. En cuanto a las variables positivas, los cuatro usuarios suben en sus niveles de CPT en el post y luego descienden en el seguimiento; en cambio, en SV, los usuarios suben progresivamente (o se mantienen) en el post y el seguimiento, a excepción del usuario Y1 que sube ligeramente en el post y luego desciende en el seguimiento a un nivel inferior al que mostraba en la evaluación pre.

Tabla 3. Estadísticos descriptivos de las variables de estudio en las evaluaciones pre, post y seguimiento

\begin{tabular}{lllllll}
\hline \hline & \multicolumn{2}{l}{ Pre-intervención } & \multicolumn{2}{l}{ Post-intervención } & \multicolumn{2}{l}{ Seguimiento } \\
\hline Variables & M & DE & M & DE & M & DE \\
\hline Síntomas Postraumáticos & 24.25 & & 17.25 & 6.65 & 14.50 & 7.68 \\
Síntomas Depresivos & 31.00 & 5.35 & 25.75 & 11.30 & 21.75 & 13.72 \\
Crecimiento Postraumático & 26.00 & 9.42 & 40.25 & 5.68 & 35.50 & 4.66 \\
Satisfacción con la Vida & 22.00 & 7.75 & 23.75 & 7.27 & 23.75 & 11.47 \\
\hline \hline
\end{tabular}


En la Tabla 3 y en la Figura 1, que representa gráficamente las medias obtenidas, se observa una reducción de los síntomas depresivos y postraumáticos, y un aumento del crecimiento postraumático y satisfacción con la vida entre las evaluaciones pre y post. Luego, se observa una nueva disminución en los puntajes de sintomatología depresiva y postraumática en el seguimiento. El crecimiento postraumático aumenta entre el pre y post y luego disminuye en el seguimiento, aunque a valores más altos que el valor pre. La satisfacción con la vida se mantiene casi sin variación entre las mediciones.

Debido a lo pequeño de la muestra, se realizaron solo análisis descriptivos.

Figura 1. Medias en pre, post y seguimiento de las variables dependientes

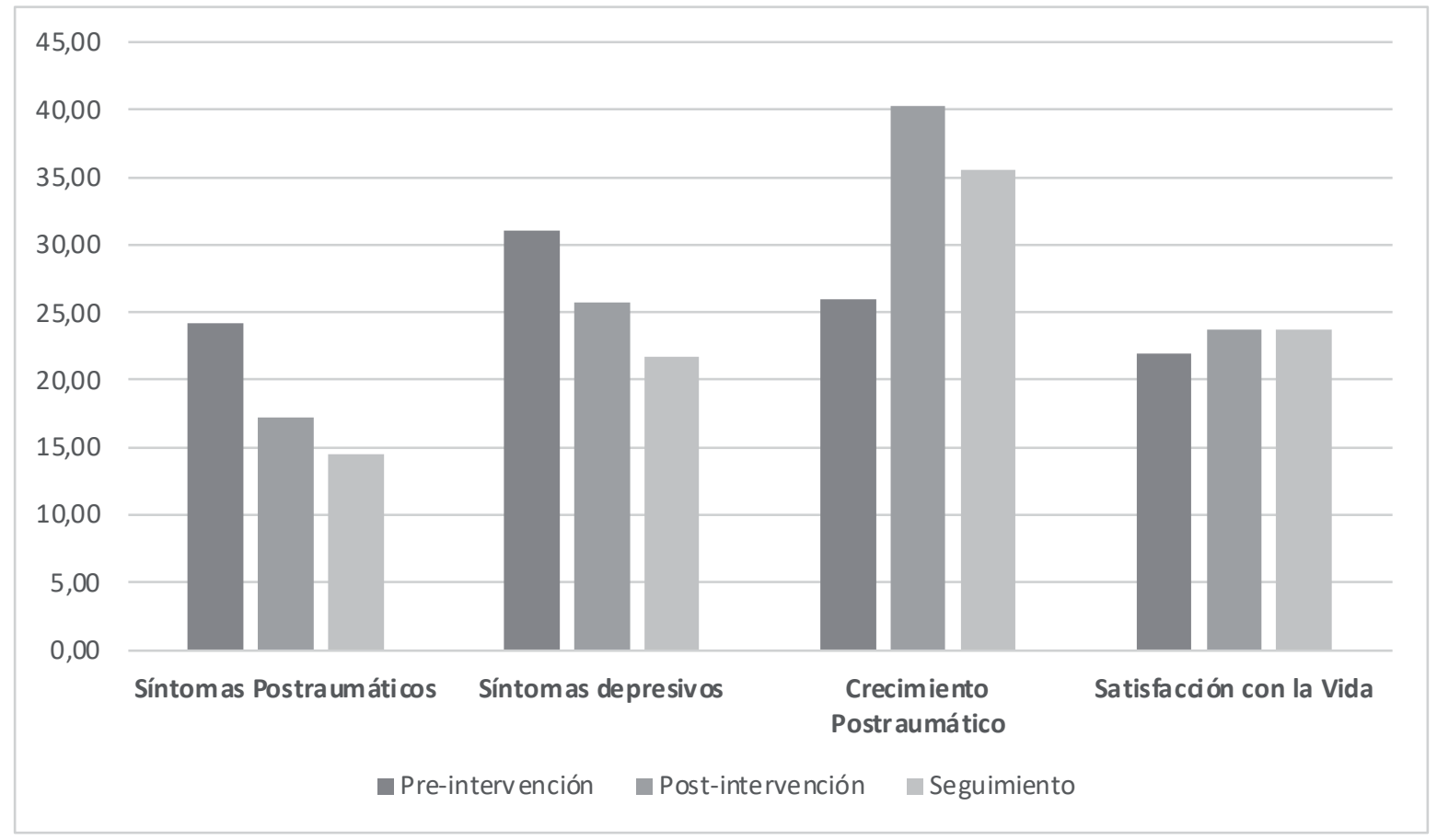

En la evaluación de la alianza terapéutica, los pun$(\mathrm{DE}=0.28 ;$ rango $=1 \mathrm{a} 10)$. En las respuestas por ítem tajes de la SRS obtuvieron una media de 9.78 por sesión observamos los resultados que se observan en la Tabla 4:

Tabla 4. Estadísticos descriptivos de las medidas de alianza terapéutica

\begin{tabular}{lll}
\hline \hline Ítem & Media & DE \\
\hline Me sentí escuchado(a), comprendido(a) y respetado(a) & 9.81 & 0.38 \\
Trabajamos y hablamos acerca de lo que yo quería trabajar o hablar & 9.75 & 0.35 \\
El método del terapeuta se adapta a mí & 9.75 & 0.20 \\
En general, la sesión de hoy estuvo bien para mí & 9.81 & 0.24 \\
Total & 9.78 & 0.28 \\
\hline \hline
\end{tabular}

\section{Discusión}

El propósito principal de este estudio fue mostrar los resultados de la intervención piloto del protocolo en Terapia Sistémica Breve, identificando la experiencia de las usuarias y terapeutas durante la intervención, así como el efecto de esta, a través de las variables dependientes de este estudio: sintomatología postraumática, 
sintomatología depresiva, crecimiento postraumático, bienestar subjetivo y alianza terapéutica.

$\mathrm{Al}$ respecto, se encontró que, si bien algunas usuarias al inicio se sentían escépticas debido a experiencias anteriores de atención psicológica, esta aprehensión se superó tras la primera sesión, pues señalaron sentirse escuchadas y en confianza, favoreciendo el vínculo emocional con su terapeuta, algo de crucial relevancia para el cumplimiento de los objetivos de una intervención (García \& Schaefer, 2015). La aplicación de una escala cuantitativa que evalúa la relación terapéutica desde la percepción del usuario reafirma esta conclusión, puesto que los puntajes se acercaron al máximo posible. La TSB entiende la alianza terapéutica como una relación de colaboración en la que compete al profesional hacer el esfuerzo de escuchar atentamente a los consultantes para poder ajustarse a sus objetivos, preferencias y lenguaje (Beyebach, 2006). Debido a ello, el protocolo incorpora muchos espacios en donde es posible conocer la experiencia de la usuaria en relación al evento estresante y se estimula explícitamente la escucha activa de parte del terapeuta.

Por otro lado, las usuarias recomendaron aumentar la cantidad de sesiones, pues consideraban insuficiente las cuatro sesiones planificadas, especialmente para el desarrollo de algunas técnicas como el árbol de la vida. Pese a ello, tanto ellas como los terapeutas reconocieron un cambio positivo al finalizar el proceso, lo que se ve respaldado por los indicadores cuantitativos que evidencian una disminución del malestar y un aumento del crecimiento postraumático. Al finalizar la terapia, las usuarias refirieron ser capaces de identificar sus recursos, sentirse más empoderadas, abrirse a otras relaciones y valorar más las cosas que las rodean. La disminución de la sintomatología postraumática y depresiva resulta relevante considerando que son los dos problemas de salud mental más frecuentes tras vivir una experiencia altamente estresante (Forbes et al., 2020; Van der Kolk, 2014). Por otro lado, el aumento en los niveles de crecimiento postraumático es explicable por la naturaleza misma del protocolo de intervención, centrado en el desarrollo de recursos y de aprendizajes a partir del evento vivido.

Dentro de las actividades desarrolladas en sesión que las usuarias destacaron como más útiles se destaca la técnica del árbol de la vida (Ncube \& Denborough,
2008), la cual les hizo recordar momentos trascendentes de su vida, reconocer fortalezas personales y recursos interpersonales y establecer metas futuras. Existe evidencia de otros estudios que han incorporado esta técnica y que han registrado una alta satisfacción por parte de los usuarios (Hirschson et al., 2017; Jorquera, 2010). Otra técnica reconocida por su utilidad fue la pregunta de escala (de Shazer, 1994), pues permitió a las usuarias observar los cambios en su estado de ánimo, expresados en números. Por último, los terapeutas destacaron la utilidad de las tareas asignadas al finalizar cada sesión, pues facilitaban el proceso de intervención y optimizaban los recursos de tiempo.

Si bien no se explicitaba en el propio protocolo, algunos terapeutas utilizaron metáforas para representar aspectos de lo que narraba la usuaria. El uso de metáforas no se escapa de los lineamientos de la TSB, por lo que su inclusión resultaba coherente con los principios y objetivos trazados en el protocolo. Las metáforas permiten capturar la atención de las usuarias, son fáciles de recordar, facilitan la comprensión, permiten introducir ideas y nuevos significados de una forma indirecta, permiten sembrar ideas entre sesiones y facilitan la externalización del problema (García \& Schaefer, 2015), por lo que su inclusión en una nueva versión del protocolo resulta relevante.

Con respecto a las limitaciones del estudio, podemos señalar lo pequeño de la muestra, que llevó a obtener apreciaciones de un número acotado de usuarios y terapeutas. Tampoco existió un grupo control que permitiera comparar resultados. Por estos motivos, los datos cuantitativos obtenidos no permiten obtener conclusiones con relación a la efectividad del protocolo. La función de este estudio piloto fue evaluar la aceptabilidad y valoración del protocolo, a fin de permitir su aplicación en un grupo más numeroso de personas en un futuro estudio experimental; en tal sentido, a pesar de sus limitaciones, el presente estudio alcanza el objetivo planteado.

En conclusión, podemos señalar que el protocolo evaluado generó apreciaciones positivas en terapeutas y usuarias, permitiendo el desarrollo de una relación terapéutica adecuada y con indicadores cuantitativos de cambio. Específicamente, se observó una disminución de la sintomatología depresiva y postraumática y un aumento del crecimiento postraumático. Asimismo, fue posible 
establecer algunos cambios en el protocolo, en la línea de flexibilizar algunas técnicas, como las tareas, y permitir la inclusión de otras, como las metáforas. De esta manera, entendemos que el protocolo está ya en condiciones de aplicarse en futuros estudios controlados.

\section{REFERENCIAS}

Agorastos, A., Marmar, C., \& Otte, C. (2011). Immediate and early behavioral interventions for the prevention of acute and posttraumatic stress disorder. Current Opinion in Psychiatry, 24(6), 526-532. https://doi.org/10.1097/ YCO.0b013e32834cdde2

Arias, P., \& García, F. (2018). Propiedades psicométricas de la Escala de Satisfacción con la Vida en población ecuatoriana adulta. Pensamiento Psicológico, 16(2), 21-29. https://doi.org/10.11144/Javerianacali.PPSI16-2.ppes

Barlow, D., \& Hersen, M. (1988). Diseños experimentales de caso único. Martínez Roca.

Beauchemin, J. (2015). Examining the effectiveness of a short-term solution-focused wellness group intervention on perceived stress and wellness among college students. [Dissertation for the Doctor Degree]. The Ohio State University, USA.

Beyebach, M. (2006). 24 ideas para una psicoterapia breve. Herder.

Beyebach, M., \& Herrero de Vega, M. (2010). 200 tareas en terapia breve individual, familiar y de parejas. Herder.

Boe, O., \& Hagen, K. (2015) Using mindfulness to reduce the perception of stress during an acute stressful situation. Procedia - Social and Behavioral Sciences, 197, 858-868. https://doi.org/10.1016/j. sbspro.2015.07.262

Bonanno, G., \& Diminich, E. (2013). Annual research review: Positive adjustment to adversity-trajectories of minimal-impact resilience and emergent resilience. Journal of Child Psychology and Psychiatry, 54(4), 378-401. https://doi.org/10.1111/ jcpp. 12021

Bonanno, G., Westphal, M., \& Mancini, A. (2011). Resilience to loss and potential trauma. Annual Review of Clinical Psychology, 7(1), 511-535. https://doi. org/10.1146/annurev-clinpsy-032210-104526

Cann, A., Calhoun, L., Tedeschi, R., Taku, K., Vishnevsky, T., Triplett, K., \& Danhauer, S. (2010). A short form of the Posttraumatic Growth Inventory. Anxiety, Stress, \& Coping, 23(2), 127-137. https://doi. org/10.1080/10615800903094273

Chen, J., Zhou, X., Zeng, M., \& Wu, X. (2015). Posttraumatic stress symptoms and post-traumatic growth: Evidence from a longitudinal study following an earthquake disaster. PLoS One, 10(6), e0127241. https://doi.org/10.1371/journal. pone. 0127241

Connor, K., \& Davidson, J. (2001). SPRINT: A brief global assessment of post-traumatic stress disorder. International Clinical Psychopharmacology, 16(5), 279-284. https://doi.org/10.1097/00004850200109000-00005

De Shazer, S. (1994). Words were originally magic. Norton.

Diener, E., Emmps, R., Larsen, R., \& Griffin, S. (1985). The satisfaction with life scale. Journal of Personality Assessment, 49(1), 71-75. https://doi.org/10.1207/ s15327752jpa4901_13

Duncan, B., Miller, S., Sparks, J, Claud, D. Reynolds, L., Brown, J., \& Johnson, L. (2003). The Session Rating Scale: Preliminary psychometric properties of a "working" alliance measure, Journal of Brief Therapy, 3(1), 3-12.

Eads, R., \& Lee, M. (2019). Solution Focused Therapy for trauma survivors: A review of the outcome literature. Journal of Solution-Focused Brief Therapy, 3(1), 47-65.

Elgueta, P., \& Fantuzzi, F. (2008). Traducción y validación chilena de la encuesta de sesión (SRS). (Tesis de Magíster). Universidad Gabriela Mistral, Santiago, Chile.

Forbes, D., Bisson, J.I., Monson, C.M., \& Berliner, L. (2020). Effective treatments for PTSD. Practice guidelines from the International Society for Traumatic Stress Studies (3rd ed.). Guilford.

García, F., Cova, F., Páez, D., \& Miranda, F. (2018). Brooding as moderator of depressive symptoms after a work accident: A longitudinal study. Scandinavian journal of psychology, 59(2), 236-242. https:// doi.org/10.1111/sjop.12433 
García, F., \& Rincón, P. P. (2011). Prevención de sintomatología postraumática en mujeres con cáncer de mama: un modelo de intervención narrativo. Terapia Psicológica, 29(2), 175-183. https://doi. org/10.4067/S0718-48082011000200004

García, F., Mardones, R., \& Ceberio, M. (2016). El proceso terapéutico en terapia sistémica breve. En F. García \& M. Ceberio (Eds.), Manual de terapia sistémica breve (pp. 113-139). Mediterráneo.

García, F., \& Schaefer, H. (2015). Manual de técnicas de psicoterapia breve: Aportes desde la terapia sistémica. Mediterráneo.

García, F., \& Wlodarczyk, A. (2015). Psychometric properties of the Posttraumatic Growth Inventory Short Form among Chilean adults. Journal of Loss and Trauma, 21 (4), 303-314. https://doi.org/10.1 080/15325024.2015.1108788

Gempp, R., Avendaño, C., \& Muñoz, K. (2004). Normas y punto de corte para la escala de depresión del centro para estudios epidemiológicos (CES-D) en población juvenil chilena. Terapia Psicológica, 22(2), 145- 156.

González, T., \& Cano, A. (2010). Introducción al análisis de datos en investigación cualitativa: Tipos de análisis y proceso de codificación (II). Nure Investigation, 45, 1-10.

Herrero de Vega, M. (2016). Trabajando la autoestima en grupo: Un programa centrado en soluciones. En F. García \& M. Ceberio (Eds.), Manual de terapia sistémica breve (pp. 381-394). Mediterráneo.

Hernández, R., Fernández, C., \& Baptista, P. (2003).Metodología de la Investigación. Mc Graw Hill.

Hirschson, S., Fritz, E., \& Kilian, D. (2017). The Tree of Life as a Metaphor for Grief in AIDS-Orphaned Adolescents. American Journal of Dance Therapy, 40(1), 87-109. https://doi.org/10.1007/s10465017-9243-7

IBM Corp (2011). IBM SPSS Statistics for Windows, Version 21.0. IBM corp.

Jorquera, P. (2010). Vejez y envejecimiento: Imaginarios sociales presentes en los textos escolares oficiales del Ministerio de educación chileno. Revista Mad, (22), 132-165. https://doi.org/10.5354/07180527.2010 .13642
Kazdin, A. E. (2001). Conduct Disorder. International Encyclopedia of the Social \& Behavioral Sciences, 2511 2516. https://doi.org/10.1016/b0-08-0430767/01349-8

Leiva-Bianchi, M., \& Gallardo, I. (2013). Validation of the Short Posttraumatic Stress Disorder Rating Interview (SPRINT-E) in a sample of people affected by F-27 Chilean earthquake and tsunami. Anales de Psicología, 29(2), 328-334. https://doi. org/10.6018/analesps.29.2.130681

Lopes, R., Gonçalves, M., Machado, P., Sinai, D., Bento, T., \& Salgado, J. (2014). Narrative therapy vs. cognitive-behavioral therapy for moderate depression: Empirical evidence from a controlled clinical trial. Psychotherapy Research, 24(6), 662-674. https://doi.org/10.1080/10503307.2013.874052

Martín-Crespo, M., \& Salamanca, A. (2007). El muestreo en la investigación cualitativa. Nure investigación, 27(4), 1-4.

Martínez, M. (2000). La investigación cualitativa etnográfica en educación. Manual teórico-práctico. Trillas.

Ncube, N., \& Denborough, D. (2008). The tree of life manual, REPPSI. Retrieved from http://www. childfund.org.au/downloads/RESOURCES/2 REPSII/Tree_Of_Life_\%28REPSII\%29.pdf

Ochoa, C., Casellas-Grau, A., Vives, J., Font, A., \& Borràs, J. M. (2017). Positive psychotherapy for distressed cancer survivors: Posttraumatic growth facilitation reduces posttraumatic stress. International Journal of Clinical and Health Psychology, 17(1), 28-37. https://doi.org/10.1016/j.ijchp.2016.09.002

QSR International (2018). Nvivo 12 Qualitative Data Analysis Software. QSR International.

Radloff, L. (1977). The CES-D scale: A self-report depression scale for research in the general population. Applied Psychological Measurement, 1(3), 385-401. https://doi.org/10.1177/014662167700100306

REPSSI (2017). Tree of Life: A workshop methodology for children, young people and adults. Baltimore, MD: Catholic Relief Services. Disponible en https://www. crs.org/sites/default/files/tools-research/tree-oflife.pdf

Sutin, A., Costa Jr, P., Wethington, E., \& Eaton, W. (2010). Turning points and lessons learned: Stressful life events and personality trait development across 
middle adulthood. Psychology and Aging, 25(3), 524-533. https://doi.org/10.1037/a0018751

Tedeschi, R., \& Calhoun, L. (1996). The Posttraumatic Growth Inventory: Measuring the positive legacy of trauma. Journal of Traumatic Stress, 9(3), 455471. https://doi.org/10.1002/jts.2490090305

Van der Kolk, B. A. (2003). The body takes the score. Mind, brain and body in the transformation of trauma. Penguin Random House.

Weakland, J., Fisch, R., Watzlawick, P., \& Bodin, A. (1974). Brief therapy: Focused problem resolution. Family Process, 13, 141-168.

White, M., \& Epston, D. (1993). Medios narrativos para fines terapéuticos. Gedisa. 